\title{
Spectral Characteristics of Hot Electron Electroluminescence in Silicon Avalanching Junctions
}

\author{
Monuko du Plessis, Senior Member, IEEE, Petrus Johannes Venter, Senior Member, IEEE, \\ and Enrico Bellotti, Associate Member, IEEE
}

\begin{abstract}
The emission spectra of avalanching $\mathbf{n}^{+} \mathbf{p}$ junctions manufactured in a standard 350-nm CMOS technology with no process modifications are measured over a broad spectral range and at different current levels. In contrast to the narrow-band forward-biased pn junction emission spectrum, the reverse biased avalanching emission spectrum extends from the ultraviolet $350 \mathrm{~nm}(3.6 \mathrm{eV})$ to the near infrared $1.7 \mu \mathrm{m}(0.7 \mathrm{eV})$, covering the visual range. The photon emission energy spectrum is compared to the hot electron energy distribution within the conduction band, as determined from a full band Monte Carlo simulation. This allows the identification of phonon assisted indirect intraband $(c-c)$ hot electron transitions as the dominant physical light emission processes within the high electric field avalanching junction. Device simulations are utilized to identify the device drift region as the source of the near infrared emissions.
\end{abstract}

Index Terms-Avalanche, electroluminescence, emission, spectrum.

\section{INTRODUCTION}

$\mathbf{T}$ HE quest for an efficient light source in silicon has been described in the past as looking for the Holy Grail of the silicon integrated circuit technology [1]. This quest is still ongoing and several approaches are followed to generate photons in the indirect band gap silicon material. These techniques include [2] dislocation-based silicon after boron implantation, silicon nanocrystals embedded in oxide, porous silicon structures, Er doping of silicon, $\mathrm{Si} / \mathrm{Ge}$ quantum dots and ion implanted $\mathrm{Si}$ in $\mathrm{SiO}_{2}$. The possibility of realizing a silicon laser has been proposed previously [1]. Since then significant progress has been made towards this aim using a variety of approaches (e.g. an optically pumped Raman siliconbased laser) for creating lasers on silicon [3]. A very interesting approach was to combine silicon nanocrystals incorporated in a silica matrix with $\mathrm{Er}^{3+}$ doping to generate light emission around $1500 \mathrm{~nm}$ in silicon, since Er-doped Si-ncs behave as electron-hole pair traps [4].

M. du Plessis and P. J. Venter are with the Carl and Emily Fuchs Institute for Microelectronics, Department of Electrical, Electronic and Computer Engineering, University of Pretoria, Pretoria 0002, South Africa (e-mail: monuko@up.ac.za; jannes.venter@up.ac.za).

E. Bellotti is with the Electrical and Computer Engineering Department, Boston University, Boston, MA 02215 USA (e-mail: bellotti@bu.edu).

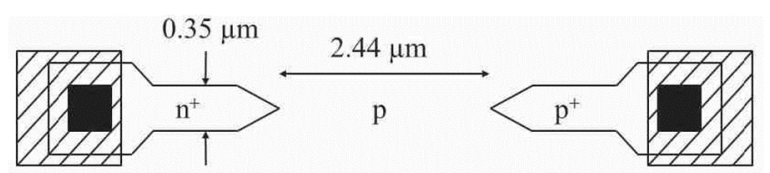

(a)

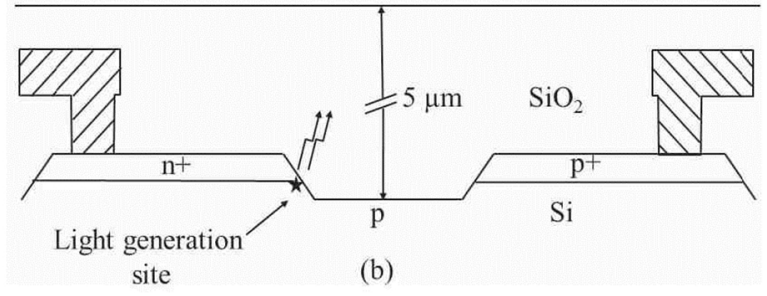

Fig. 1. (a) Layout design of CMOS $n^{+}$p point source, and (b) cross section of $n^{+} p$ point source. Light generation takes place along the sidewall of the LOCOS (local oxidation) structure.

However, the key to successful commercialization of any silicon-based light source technology will be to develop a high-volume manufacturing process where light sources can be processed in a CMOS fabrication facility alongside existing CMOS wafers in order to amortize their costs [5]. CMOS compatibility is of the utmost performance in order to take advantage of the vast investment in current CMOS fabrication facilities. All of the above-mentioned technological approaches to manufacture silicon-based light sources necessitate modifications to existing CMOS processes.

Our approach is quite different, namely investigating the silicon light source devices already present in state-of-the-art CMOS technologies, thus eliminating the CMOS compatible issue completely since our devices are $100 \%$ CMOS with no process modifications [6]. Specifically, we are investigating the electroluminescence of hot electrons under high electric field conditions. CMOS based light sources may find a wide range of applications if adequate levels of internal quantum efficiency and external power efficiency can be reached. Applications already proposed and demonstrated include micro displays [7], [8] short haul optical data links [9], [10] and lab-on-chip systems [11], [12]. Although the phenomenon of light emission from a silicon avalanching junction has been known for more than 57 years [13], the physical light generation mechanisms have been studied in more detail only 

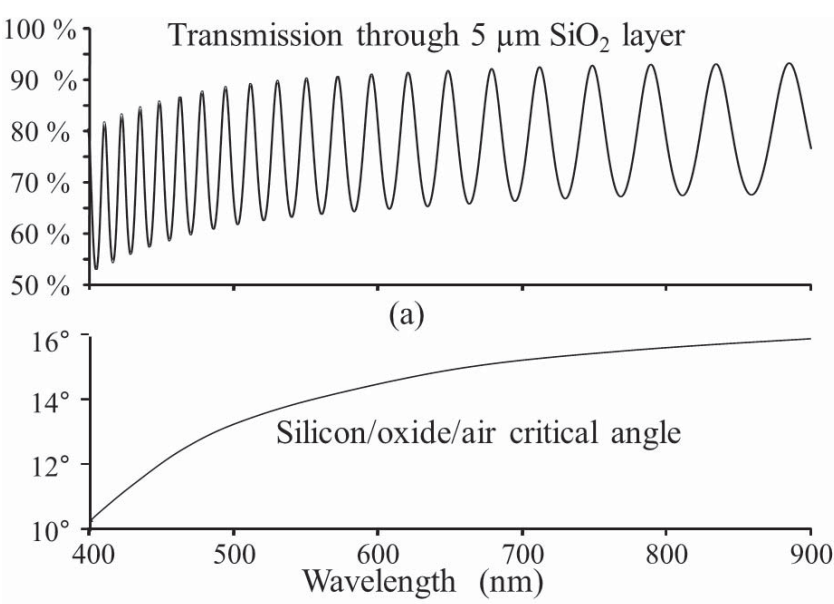

(b)

Fig. 2. (a) Calculated transmission of radiation through the $5 \mu \mathrm{m}$ thick silicon dioxide layer, and (b) the calculated critical angle for total internal reflection in the silicon.

during the last two decades. Even today, the dominant physical mechanisms responsible for photon generation in avalanching indirect band gap semiconductor junctions are not well known and/or generally accepted. In this paper we will use wideband spectral measurements, combined with Monte Carlo device simulations and one dimensional device simulations to identify the dominant radiative processes.

\section{Methodology}

\section{A. Device Design}

The pn junctions under investigation were designed and manufactured in the standard ams $0.35 \mu \mathrm{m}$ CMOS foundry process without any post-processing. The $\mathrm{n}^{+}$to $\mathrm{p}$-substrate junction was chosen as the junction of interest, and the layout was performed with the aim to achieve as high an electric field as possible, as will be discussed later. The layout and cross section of a single point source test device are shown in Fig. 1. The $\mathrm{n}^{+}$in p-substrate junction forms a sharp tip, pointing towards a $\mathrm{p}^{+}$substrate contact, to increase both the electric field and current density when avalanching takes place. Care was taken with the layout to prevent light being reflected from or shielded by any metal layers in close proximity. To achieve higher levels of optical power being emitted, 400 identical point sources were connected in parallel to form an array. The nominal test current was $140 \mathrm{~mA}$, with $350 \mu \mathrm{A}$ flowing in each point source at $10.5 \mathrm{~V}$ reverse bias. An important design consideration when designing pn junctions in avalanche breakdown is the current density in the device. At $140 \mathrm{~mA}$, and with the junction depth of $0.2 \mu \mathrm{m}$, the maximum current density in the pn junction is determined as $500 \mathrm{kA} / \mathrm{cm}^{2}$. This sounds quite high, but when compared to the current densities in the NMOS transistors, it is still within limits. The maximum saturated "on drive current" density measured in the NMOS transistors with $3.3 \mathrm{~V}$ bias on gate and drain is $600 \mu \mathrm{A} / \mu \mathrm{m}$ width as specified by the manufacturing foundry, which corresponds to a maximum "on" drain current of $210 \mu \mathrm{A} / 0.35 \mu \mathrm{m}$ width. This shows that the maximum

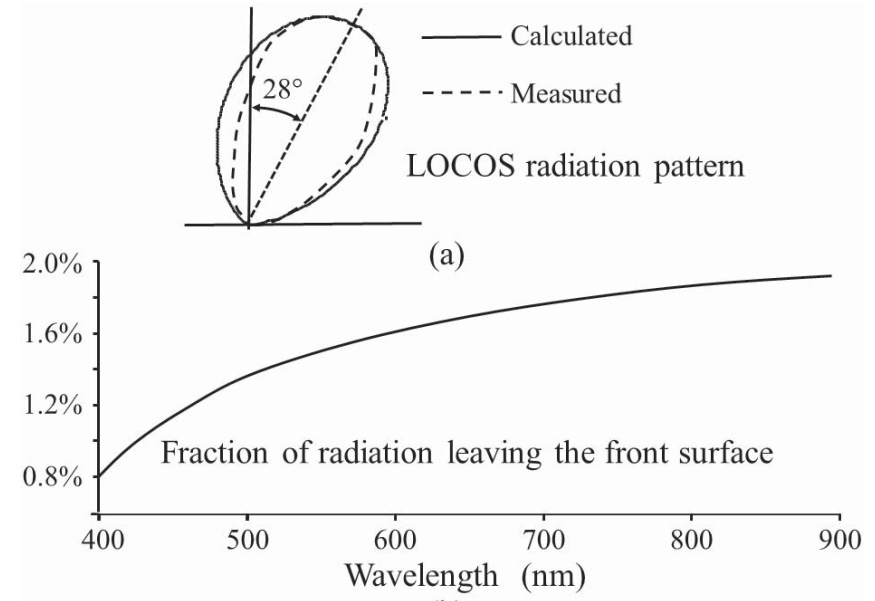

(b)

Fig. 3. (a) Measured and calculated radiation pattern, and (b) the fraction of total light being generated that will leave the front surface of the device.

reverse bias current of $350 \mu \mathrm{A}$ per point source is only $67 \%$ higher than that experienced in the NMOS transistor drain/source region under normal operating conditions.

\section{B. Spectral Measurements}

The output spectra of the array of 400 point sources were measured using two different spectrometers, namely 1) a Si UV-Vis-NIR spectrometer (Avantes AvaSpec 2048TEC) covering the range $250 \mathrm{~nm}$ to $1100 \mathrm{~nm}$, and 2) an InGaAs NIR spectrometer (B\&WTEK SOL 1.7) covering the wavelength range $900 \mathrm{~nm}$ to $1700 \mathrm{~nm}$. From the known relative responses of the two spectrometers, as well as their overlapping spectral ranges, the two spectra could be merged into one externally observed spectrum. From these measured spectra, as well optical power measurements from an International Light Radiometer (IL1700) power meter using a silicon detector SED100 covering the range $250 \mathrm{~nm}$ to $1100 \mathrm{~nm}$, and a InGaAs detector SED007 covering the range $800 \mathrm{~nm}$ tot $1600 \mathrm{~nm}$, the photon emission rates at the light generation site within the silicon bulk could be determined. Aspects to consider were the transmission through the thick oxide layer, as well as the effect of the critical angle for total internal reflection within the silicon. The calculated spectral characteristics of the oxide transmission and the silicon critical angle are illustrated in Fig. 2.

In Fig. 2 the effect of the increase of the silicon refractive index at shorter wavelengths can be seen. Also very evident is the interference patterns of transmission through a relatively thick oxide layer. It is important to note that a smaller critical angle at shorter wavelengths will lead to less radiation leaving the front surface of the device. Fig. 3 shows the calculated and measured radiation pattern, as well as the fraction of light leaving the front surface as a function of wavelength.

The measured radiation pattern was slightly narrower than the calculated one, and the peak radiation at an angle of $28^{\circ}$ from the vertical corresponds to a $45^{\circ}$ angle of the LOCOS sidewall. Taking all of the effects into account it follows from Fig. 3(b) that at $400 \mathrm{~nm}$ only $0.8 \%$ of the generated light will leave the front surface. This value increases to about $2 \%$ at 


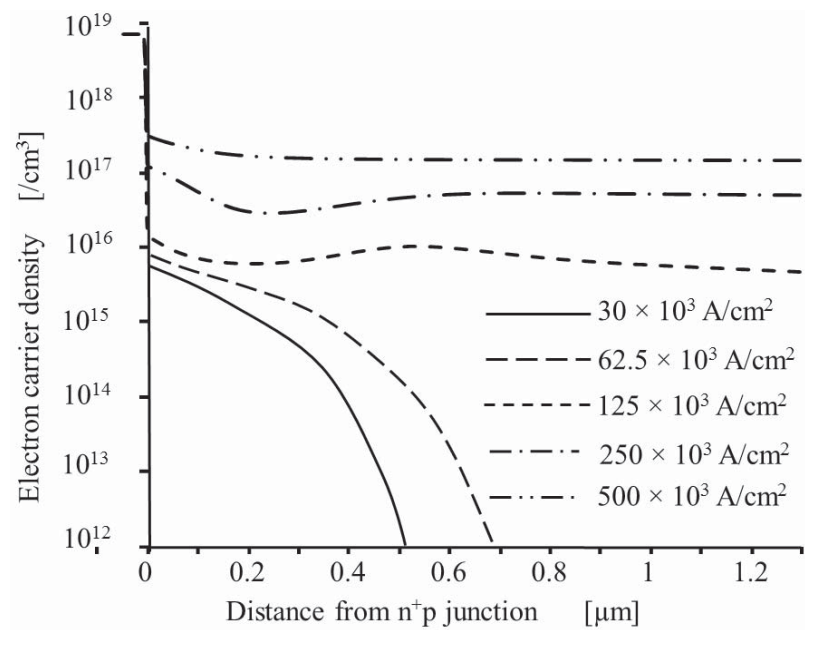

Fig. 12. The simulated electron carrier density distribution in the device at different current densities. Below $100 \times 10^{3} \mathrm{~A} / \mathrm{cm}^{2}$ the electron concentration in the drift region (distance from junction $>0.8 \mu \mathrm{m}$ ) is very low. This threshold current density of $100 \times 10^{3} \mathrm{~A} / \mathrm{cm}^{2}$ will be a function of the acceptor doping in the p-type drift region as well as of the device geometry.

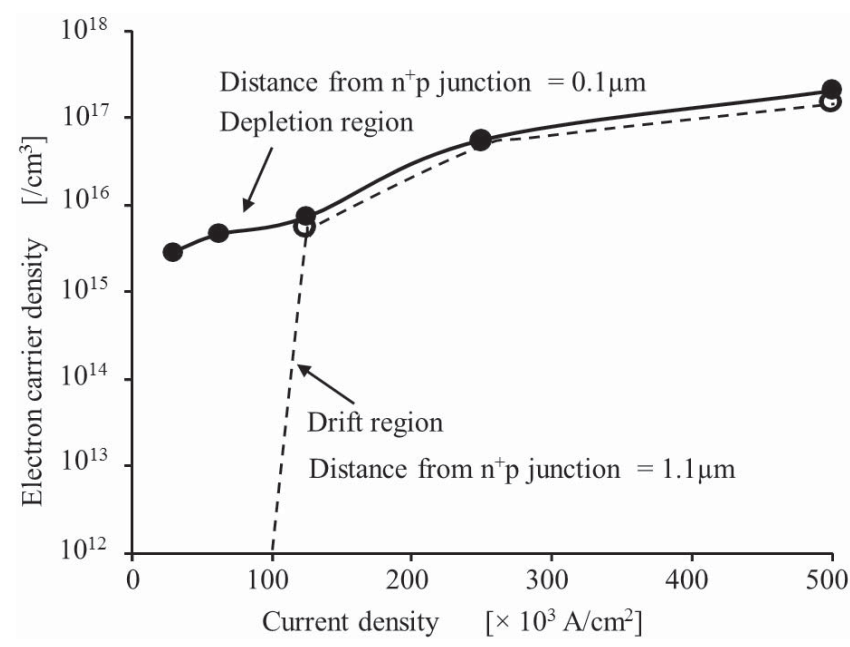

Fig. 13. The electron carrier density in the depletion and drift regions of the device at different current densities. At a current density of $100 \times 10^{3} \mathrm{~A} / \mathrm{cm}^{2}$ the electron concentration in the drift region falls to a very small value. The correlation between this figure and the measured photon emission shown in Fig. 9 is observed.

In Fig. 12 it is shown that in the drift region the electron carrier density is small for current densities less than $100 \times 10^{3} \mathrm{~A} / \mathrm{cm}^{2}$. For current densities higher than this value, the electron carrier densities are basically the same in both the depletion and drift region. Again the variation of electron density with current density at a constant distance from the $\mathrm{n}^{+} \mathrm{p}$ interface can be derived. This result is shown in Fig. 13 for distances $0.1 \mu \mathrm{m}$ (depletion region) and $1.1 \mu \mathrm{m}$ (drift region) from the interface. From Fig. 13 it can be seen that the electron carrier density increases with increasing current density, as expected. The electron density in the drift region is very similar to that of the depletion region for current densities above $100 \times 10^{3} \mathrm{~A} / \mathrm{cm}^{2}$, but below this value of current density the electron density drops suddenly to a very low value.

\section{INTERPRETATION OF RESULTS}

From the measured spectrum (Fig. 8) and the photon energy "signature" of the second peak in the DOS function, we identify indirect intraband hot electron $c-c$ transitions as the main light generating mechanism. This is in line with an analysis performed recently on similar devices [27].

The photon emissions from the high electric field depletion region of the device emits a spectrum (see Fig. 8) that correlates well with the $600 \mathrm{kV} / \mathrm{cm}$ electron energy distribution (see Fig. 5). Emission from the low electric field drift region only becomes noticeable above a current density of about $100 \times 10^{3} \mathrm{~A} / \mathrm{cm}^{2}$ when the electric field increases rapidly to a value of about $200 \mathrm{kV} / \mathrm{cm}$ (see Figs. 9, 11, and 13), where it remains for larger current densities. This photon emission from the drift region is also due to indirect intraband hot electron $c-c$ transitions, since the low photon energy drift region emission spectrum correlates very well with the $200 \mathrm{kV} / \mathrm{cm}$ electron energy distribution (see Fig. 5). It then follows that the photon emission spectra of Fig. 8 at high current density constitutes the combined effect of the $200 \mathrm{kV} / \mathrm{cm}$ and $600 \mathrm{kV} / \mathrm{cm}$ electron energy distributions shown in Fig. 5. At low current densities in the drift region, only the $600 \mathrm{kV} / \mathrm{cm}$ spectrum from the depletion region will be observed. It can thus be concluded that the two spectra $(<1.15 \mathrm{eV}$ and $>1.15 \mathrm{eV})$ observed in Fig. 8 emanate from two different regions in the device, namely the high electric field depletion region $(>1.15 \mathrm{eV})$ and the low electric field drift region $(<1.15 \mathrm{eV})$. Since the drift region geometry and doping levels will be a strong function of the device design and process technology, the threshold level of reverse current to initiate low photon energy emissions from the drift region may vary significantly from our observed $70-100 \times 10^{3} \mathrm{~A} / \mathrm{cm}^{2}$.

\section{ACKNOWLEDGMENT}

The authors would like to thank Christo Janse van Rensburg and Alfons Bogalecki (INSiAVA (Pty) Ltd., Pretoria, South Africa) for performing the measurements. We also would like to thank Hanqing Wen (Boston University, Boston MA, USA) for the device simulations.

\section{REFERENCES}

[1] N .R. Savage, "Holy grail: Light from silicon," in Proc. IEEE Spectr. Conf., Jan. 2004, pp. 51-59.

[2] L. Pavesi, S. Gaponenko, and L. Dal Negro, Nato Science Series II: Mathematics, Physics and Chemistry Series, vol. 93, Boston, MA, USA: Kluwer Academic Publishers, 2003.

[3] D. Liang and J. E. Bowers, "Recent progress in lasers on silicon," Nature Photon., vol. 4, no. 8, pp. 511-517, 2010.

[4] C. Ciminelli, P. Frascella, and M. N. Armenise, "Optical modelling of a Si-based DBR laser source using a nanocrystal Si-sensitized Er-doped silica rib waveguide in the C-band," J. Eur. Opt. Soc. Rapid Publications, vol. 3, pp. 08017-1-08017-6, Jan. 2008.

[5] N. Izhaky, M. T. Morse, S. Koehl, O. Cohen, D. Rubin, A. Barkai, G. Sarid, R. Cohen, and M. J. Paniccia, "Development of CMOScompatible integrated silicon photonic devices," IEEE J. Sel. Topics Quantum Electron., vol. 12, no. 6, pp. 1688-1698, Nov.-Dec. 2006.

[6] M. du Plessis, H. Aharoni, and L. W. Snyman, "Silicon LEDs fabricated in standard VLSI technology as components for all silicon monolithic integrated optoelectronic systems," IEEE J. Sel. Topics Quantum Electron., vol. 8, no. 6, pp. 1412-1419, Nov.-Dec. 2002.

[7] P. J. Venter, M. du Plessis, A. W. Bogalecki, M. E. Goosen, and P. Rademeyer, "An $8 \times 64$ pixel dot matrix microdisplay in 0.35 micron CMOS technology," Opt. Eng., vol. 51, no. 1, p. 014003, 2012. 
[8] A. R. Chen, A. I. Akinwande, and H.-S. Lee, "CMOS-based microdisplay with calibrated backplane," IEEE J. Solid-State Circuits, vol. 40, no. 12, pp. 2746-2755, Dec. 2005.

[9] A. Chatterjee, P. Mongkolkachit, B. Bhuva, and A. Verma, "All Si-based optical interconnect for interchip signal transmission," IEEE Phot. Tech. Lett., vol. 15, no. 11, pp. 1663-1665, Nov. 2003.

[10] S. Sayil, "Avalanche breakdown in silicon devices for contactless logic testing and optical interconnect," Analog Integr. Circuit Signal Process., vol. 56, no. 3, pp. 213-221, 2008.

[11] L. Rebohle, T. Gebel, R. A. Yankov, T. Trautmann, W. Skorupa, J. Sun, G. Gauglitz, and R. Frank, "Microarrays of silicon-based light emitters for novel biosensor and lab-on-a-chip applications," Opt. Mater., vol. 27, no. 5, pp. 1055-1058, 2005.

[12] K. Misiakos, P. S. Petrou, S. E. Kakabakos, M. E. Vlahopoulou, A. Tserepi, E. Gogolides, and H. H. Ruf, "Monolithic silicon optoelectronic transducers and elastomeric fluidic modules for bio-spotting and bio-assay experiments," Microelectron. Eng., vol. 83, nos. 4-9, pp. 1605-1608, 2006.

[13] R. Newman, W. C. Dash, R. N. Hall, and W. E. Burch, "Visible light from a Si p-n junction," Phys. Rev., vol. 98, no. 1, pp. 1536-1537, 1955

[14] L. Tirino, M. Weber, K. F. Brennan, and E. Bellotti, "A general Monte Carlo model including the effect of the acoustic deformation potential on the transport properties," J. Comput. Electron., vol. 3, no. 2, pp. 81-93, 2005.

[15] M. V. Fischetti, "Monte Carlo simulation of transport in technologically significant semiconductors of the diamond and zinc-blende structuresPart I: Homogeneous transport," IEEE Trans. Electron Devices, vol. 38, no. 3, pp. 634-649, Mar. 1991.

[16] R. Brunetti, C. Jacoboni, F. Venturi, E. Sangiorgi, and B. Riccò, "A many-band silicon model for hot-electron transport at high energies," Solid-State Electron., vol. 32, no. 12, pp. 1663-1667, 1989.

[17] T. Mietzner, J. Jakumeit, and U. Ravaioli, "Local iterative Monte Carlo analysis of electron-electron interaction in short-channel SiMOSFETs," IEEE Trans. Electron Devices, vol. 48, no. 10, pp. 2323-2330, Oct. 2001.

[18] A. G. Chynoweth and K. G. McKay, "Photon emission from avalanche breakdown in silicon," Phys. Rev., vol. 102, no. 2, pp. 369-376, 1956.

[19] A. L. Lacaita, F. Zappa, S. Bigliardi, and M. Manfredi, "On the Bremsstrahlung origin of hot-carrier-induced photons in silicon devices," IEEE Trans. Electron Devices, vol. 40, no. 3, pp. 577-582, Mar. 1993.

[20] N. Akil, S. E. Kerns, D. V. Kerns, A. Hoffmann, and J.-P. Charles, "A multimechanism model for photon generation by silicon junctions in avalanche breakdown," IEEE Trans. Electron Devices, vol. 46, no. 5, pp. 1022-1028, May 1999.

[21] A. G. Chynoweth and H. K. Gummel, "Photon emission from avalanche breakdown in germanium p-n junctions," J. Phys. Chem. Solids, vol. 16, nos. 3-4, pp. 191-197, 1960.

[22] G. Deboy and J. Kölzer, "Fundamentals of light emission from silicon devices," Semicond. Sci. Technol., vol. 9, no. 5, pp. 1017-1032, 1993.

[23] L. Selmi, H. S. Wong, M. Lanzoni, E. Sangiorgi, and M. Manfredi, "Investigation of hot electron luminescence in silicon by means of dual gate MOS structures," in Proc. IEDM Tech. Dig., 1993, pp. 531-534.

[24] N. Asli, M. I. Vexler, A. F. Shulekin, P. D. Yoder, I. V. Grekhov, and P. Seegebrecht, "Threshold energies in the light emission characteristics of silicon MOS tunnel diodes," Microelectron. Rel., vol. 41, no. 7, pp. 1071-1076, 2001.

[25] M. du Plessis and P. Rademeyer, "Novel electroluminescence technique to analyze mixed reverse breakdown phenomena in silicon diodes," Solid State Electron., vol. 54, no. 4, pp. 433-438, 2010.

[26] (Jun. 2012). Sentaurus Device User Guide [Online]. Available: www.synopsys.com

[27] H. Elghazi, A. Jorio, and I. Zorkani, "Analysis of silicon light emission under breakdown condition using an indirect intraband model," Opt. Commun., vol. 281, no. 12, pp. 3320-3323, 2008.
Monuko du Plessis (M'76-SM'81) was born in King Williamstown, South Africa, in 1949. He received the B.Eng., M.Eng., and the D.Eng. degrees in electrical engineering from the University of Pretoria, Pretoria, South Africa, in 1972, 1978 and 1984, respectively, and the B.A. degree in psychology and B.Com. (Hons) degree in economics from the University of South Africa, Pretoria 0002, South Africa, in 1989 and 1998, respectively.

He joined the Department of Electrical Engineering, University of Pretoria, as Research Officer in microelectronics and optoelectronics. He was the Director of the Carl and Emily Fuchs Institute for Microelectronics (CEFIM), University of Pretoria from 1991 to 2012, and is currently a Full Professor with the Department of Electrical, Electronic and Computer Engineering, University of Pretoria. His current research interests include silicon photonics and analog CMOS integrated circuit design.

Prof. Plessis is a member of SPIE and a Registered Professional Engineer with the Engineering Council of South Africa (ECSA). He is also an IEEE ED Society Distinguished Lecturer in Region 8.

Petrus J. Venter (M'04-SM'13) received the B.Eng. degree in electronic engineering in 2004 and the M.Eng. degree in 2009 from the University of Pretoria, Pretoria, South Africa. He is currently pursuing the Ph.D. degree in silicon light emission.

He was an Assistant Lecturer with the University of Pretoria from 2005 to 2006. In 2007, he was a Lecturer and started to practice engineering in a start-up company called INSiAVA founded in the same year. He is currently an Senior Lecturer Research Engineer with the University of Pretoria.

Mr. Venter was a recipient of the SAMES Award for Best Microelectronic Student in 2004 and a first prize in the Design Automation Conference's student paper contest in 2005. In 2006, he was awarded the Tanner Award for Outstanding Achievement in Educational Design.

Enrico Bellotti (A'11) received the "Laurea in Ingegneria Electtronica" degree from Politecnico di Milano, Milano, Italy, in 1989, and the Ph.D. degree in electrical engineering from the Georgia Institute of Technology, Atlanta, GA, USA, in 1999. He has been with the Electrical and Computer Engineering Department, Boston University, Boston, MA, USA, since September 2000, initially as an Assistant Professor from 2000 to 2006 then with the rank of Associate Professor from 2006 to 2012), and a Professor since 2013.

$\mathrm{He}$ has over 20 years of experience in electronic and optoelectronic device simulation, and computational electronics research. He has authored over 75 journal papers, 60 conference papers, and 4 book chapters. He hods two U.S. patents. He has done research on InGaAs/InP and AlGaN/GaN HEMTs design, GaN-based permeable base transistors, near-infrared detectors, avalanche photodiodes, high-field transport study in wide band-gap semiconductors, UV optoelectronics devices, and multispectral IR detectors. His current research interests include the microscopic simulation of terahertz emitters, avalanche and single-photon detectors, oxide semiconductor materials, and electromagnetic simulation of 3-D synthetic structures intended for emitter/detector performance enhancement.

Dr. Bellotti was a recipient of the 2003 ONR Young Investigator Program Award and the 2005 NSF CAREER Award. 